\title{
The Moroccan dialect version of the Insomnia Severity Index
}

\author{
Bouchra Oneib ${ }^{1 *}$, Abdelilah El Filali ${ }^{1}$ and Naima Abda ${ }^{2}$
}

\begin{abstract}
Background: The objective of the present study was to translate and linguistically validate in Moroccan dialect the original English version of the Insomnia Severity Index (ISI). The translation and adaptation of this scale are obtained by using the process forward translations and backward translations. The participants filled out the final questionnaire two times with an interval of 10 days. Cronbach's alpha coefficient assesses the acceptability and internal reliability. As for the test-retest reproducibility, we used the inter-class correlation coefficient (ICC) (95\% confidence interval (CI)) and the kappa coefficient, as well as the convergent validity for each scale.

Results: The sample included 120 subjects, 49 men (40.8\%) and 71 women (59.2\%). The mean age of participants was $29.23 \pm 6.69$ years. The translated version was quite acceptable. The Cronbach's alpha coefficient was 0.83 . The $\mathrm{CCl}(95 \% \mathrm{Cl})$ was $0.99(0.989,0.995)$, while the kappa coefficient was 0.76 . The correlations between the items of each questionnaire and its score showed good to strong positive correlations.

Conclusions: These results concluded that psychometric properties are adequate compared to the original version. Thus, this translated version had to be used for the sleep assessment in the Moroccan population.
\end{abstract}

Keywords: Sleep, Insomnia, Insomnia Severity Index, Moroccan dialect

\section{Background}

Insomnia is the most prevalent sleep complaint, but remains largely an unidentified public health issue.

The DSM-5 defines insomnia as a sleep disturbance with predominant dissatisfaction (sleep quantity or quality). This sleep disorder includes symptoms such as difficulty initiating sleep, difficulty maintaining sleep, and early awakening with the inability to return to sleep. Also, the main criterion to diagnosis this disorder is significant distress in social, occupational, educational, academic, behavioral, or other important areas of functioning [1].

Insomnia is the most common sleep disorder, known to be a public health issue. According to the DSM criteria, the prevalence of insomnia in the general population is

\footnotetext{
*Correspondence: boucha82@hotmail.com

1 Department of Psychiatry, CHU Mohammed VI, Faculty of Medicine, University Mohammed I, Hopital Arrazi, Boulevard Allal El Fassi, 60000 Oujda, Morocco

Full list of author information is available at the end of the article
}

about $30 \%$ [2]. We can detect insomnia disturbance by scales such as ISI.

In addition to the present adequate psychometric properties, ISI is likely to be clinically useful for several reasons. It is short with a format that is easy to administer in less than 5 min and whose score can be measured in less than $1 \mathrm{~min}$. It provides relevant information for diagnosis and treatment planning. It can guide the clinician in deciding whether an insomnia complaint reaches the clinical/diagnostic threshold. Finally, this measure evaluates the results of treatment, whether in clinical practice or research.

This instrument is designed to measure the perception of insomnia by the patient. It assesses the severity of the night and day components of insomnia. The ISI is a 7-item self-report questionnaire assessing the nature, severity, and impact of insomnia.

In this assessment, we look for insomnia during the last 2 weeks. We evaluate the severity of falling asleep, maintenance of sleep, problems waking up early in the 
morning, sleep dissatisfaction, the daily functioning, the others' perceptibility of sleep problems, and the distress caused by difficulty sleeping.

A 5-point Likert scale is used to score each item $(0=$ no problem, $4=$ very serious problem), which gives a total score ranging from 0 to 28 . The total score is interpreted as follows: absence of insomnia (0-7), subthreshold insomnia (8-14), moderate insomnia (15-21), and severe insomnia (22-28) [3].

This scale was translated into several languages, including classical Arabic [4-6]. In Morocco, the official and formal language is classical Arabic, but the Moroccan dialect remains the mother language spoken and understood by all Moroccans of various education levels and illiterate. Its everyday oral communication includes words in Arabic and Berber.

According to our knowledge, ISI does not exist in a translated version adapted to our Moroccan context. So, the objective of our study is to translate and validate this measure into the Moroccan Arabic dialect.

\section{Methods}

Before starting the translation, we obtained the authors' agreement of the scale.

\section{Translation (Fig. 1)}

These scales are translated and culturally adapted to the Moroccan dialect following the guidelines suggested by Guillemin et al. [7]. Four main steps were taken: forward translation, back translation, committee review, and pretest.

\section{First step}

The translation of the original English version of the scales was done independently by two translators fluent in English.

The two translators carried out a cross-cultural adaptation of the concepts rather than a simple linguistic translation.

The translation coordinator produced a synthesis of the two translated versions, with a detailed report of the difficulties and resolutions.

At the end of this step, a preliminary Moroccan Arabic version of the three scales is obtained.

\section{Second step}

The preliminary version was then back-translated into English by two bilingual translators. They are unfamiliar with the original English version.

The coordinator accomplished the synthesis of the two back-translated versions with a detailed report of the difficulties of back-translation.

\section{Third step}

A committee of experts, including translators and psychiatrists, revised this process, and they discussed the semantic equivalence to obtain a pre-final version.

\section{Fourth step}

Pre-test To get the final version, we test the pre-final version on adult subjects. This process helps us to eliminate any ambiguity and to check the understanding of the different items. The test was carried out on ten individuals belonging to different social categories and educational levels.

The final version was subjected to psychometric analysis.

\section{Validation \\ Sample size}

We determined the sample size on the Streiner curve, which estimates the number of subjects needed according to the desired reliability coefficient and degree of precision. For an ICC of 0.70 and an accuracy of \pm 0.10 , the number needed is approximately 120 subjects [8].

\section{Study population}

We selected 120 medical students, medical staff, and administrators working at university hospital centers. We can easily administer the scale in two steps for this population. Also, this community is more likely to have sleep disorders.

The following are the inclusion criteria:

- Participants speak the Moroccan dialect, aged over 18 years old.

- All participants were informed about the study. We respect the anonymity and confidentiality of participants during the collection of data.

The following are the exclusion criteria:

- Those who do not speak the Moroccan dialect

- Subjects with an intellectual disability or a medical condition making it difficult to understand the questionnaire

- Subjects who did not consent to participate in the study

\section{Data collection}

Data collected We carried out a self-administered nonanonymous questionnaire in the Moroccan dialect. It 


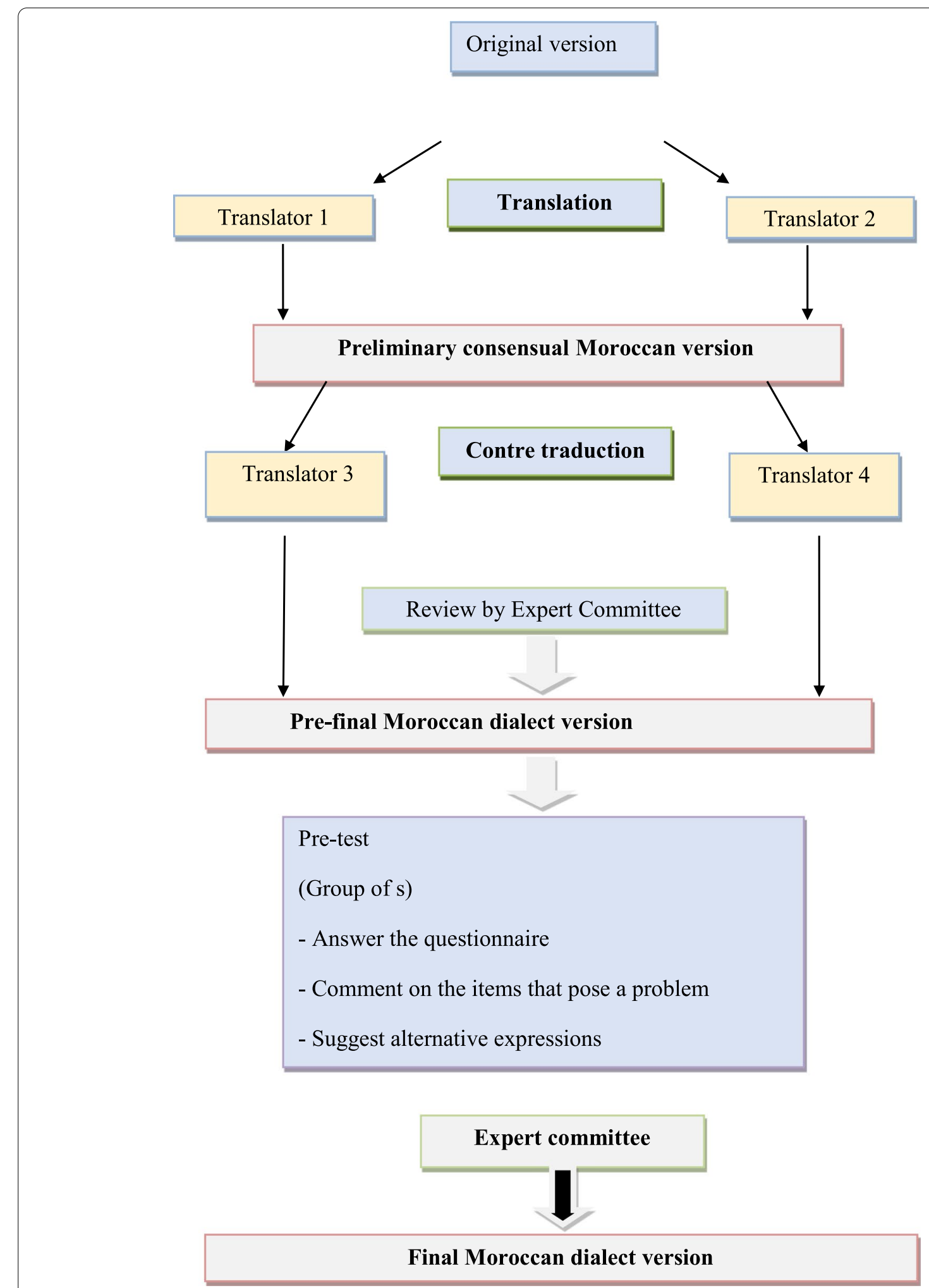

Fig. 1 Stages of transcultural adaptation to the Moroccan dialect of the ISI, ESE, and IQSP scales 
contained the socio-demographic characteristics (age, sex, marital status, profession, and socio-economic level) and the Moroccan version of the scale.

We had to pass the scales two times (T1, T2) to each individual in the sample.

We obtained oral and written consent from each participant before starting the study.

Collection methods The translated versions were administered two times by two investigators. The second administration made 10 days after the first.

\section{Ethical aspects}

The Ethics Research Committees of the Faculty of Medicine and Pharmacy Oujda approved the study. The study was carried out under conditions that comply with the ethical recommendations of the Helsinki Declaration. We obtained signed consent from participants.

\section{Data analysis}

Descriptive analysis Descriptive statistics were generated to summarize the demographic characteristics of the participants and to evaluate the score distribution. Quantitative variables were described using means, standard deviations, and range of scores. Qualitative variables were expressed as percentages.

Psychometric properties The reliability was estimated by measuring the internal consistency and the test-retest reliability of the questionnaire. The internal consistency of the Moroccan Arabic versions of the ISI scale was assessed for the multi-item questionnaire scales using Cronbach's coefficients. Internal consistency of a magnitude of 0.70 or greater was sought. Test-retest reliability was estimated by calculating the ICC derived from a two-factor analysis of variance with a random effect [9]. A correlation greater than 0.60 is usually desirable, and values greater than 0.90 indicate excellent reliability [10]. Besides, we calculated the kappa coefficient, with a value close to 1 reflects a high level of agreement $[11,12]$.

The contribution of each item to the total score was assessed using item-total correlation. Corrected itemtotal correlation $\geq 0.3$ are considered acceptable [13].

Since there was neither a relevant criterion-related estimator of prediction nor a well-defined domain of content for determining validity, construct validity should also be obtained in future studies to further verify the psychometric properties of this version.

Statistical analysis of the study population was performed using the SPSS 21.0 software.

\section{Results}

\section{Acceptability}

We note the good acceptability of the scale. Indeed, the average time required to complete them was $10 \mathrm{~min}$, and the refusal rate was $0 \%$. There was no missing answer to the different items.

\section{Sociodemographic characteristics of the sample}

One hundred twenty individuals participated in the study, with a mean age of $29.23 \pm 6.69$ years. The majority of participants were females $(59.2 \%)$ and singles $(60 \%)$ (Table 1).

All the participants were reviewed for the second test (retest).

\section{Description of items and scale score}

The means of the different items of the ISI scale ranged from 0.92 "item 2" to 2.06 "item 3." Those for T1 and T2 scores were 10.15 and 9.73, respectively (Table 2).

According to the ISI scale, only $0.8 \%$ had severe insomnia, while $45.8 \%$ presented Subthreshold insomnia (Table 3).

\section{Reliability}

Homogeneity and consistency

A Cronbach's alpha coefficient of 0.81 and 0.83 was obtained in the test and retest, respectively, for the whole scale (Table 4).

Table 1 Sociodemographic characteristics of sample $(n=120)$

\begin{tabular}{ll}
\hline Characteristic & $\boldsymbol{N}(\%)$ \\
\hline Age (years) & a \\
Gender & $29.23 \pm 6.69$ \\
$\quad$ Man & $49(40.8)$ \\
$\quad$ Woman & $71(59.2)$ \\
Marital status & \\
$\quad$ Single & $72(60.0)$ \\
$\quad$ Married & $48(40.0)$ \\
Socio-economic level & \\
$\quad$ Low & $1(0.8)$ \\
Medium & $114(95.0)$ \\
High & $5(4.2)$ \\
\hline
\end{tabular}

${ }^{\mathrm{a}}$ Means \pm standard deviation 
Table 2 Central tendency and variability of the ISI scale

\begin{tabular}{|c|c|c|c|c|}
\hline ESS items & Mean (SD) & Median (min-max) & $\begin{array}{l}\text { Floor } \\
\text { effect }^{\mathrm{a}} \\
(\%)\end{array}$ & $\begin{array}{l}\text { Ceiling } \\
\text { effect }^{\mathrm{b}} \\
(\%)\end{array}$ \\
\hline Item 1 & $1.33(1.02)$ & $1(0-4)$ & 22.5 & 1.7 \\
\hline Item 2 & $0.92(0.91)$ & $1(0-4)$ & 39.2 & 0.8 \\
\hline Item 3 & $2.06(1.34)$ & $2(0-4)$ & 15.8 & 18.3 \\
\hline Item 4 & $1.93(1.01)$ & $2(0-4)$ & 5.0 & 6.7 \\
\hline Item 5 & $1.01(0.96)$ & $1(0-4)$ & 35.8 & 1.7 \\
\hline Item 6 & $1.33(1.05)$ & $1(0-4)$ & 25.0 & 1.7 \\
\hline Item 7 & $1.64(0.99)$ & $2(0-4)$ & 10.0 & 4.2 \\
\hline Total score & $10.15(5.02)$ & $10.5(0-28)$ & 2.5 & 0.8 \\
\hline
\end{tabular}

$S D$ standard deviation

a Percentage of the lowest modality

${ }^{\mathrm{b}}$ Percentage of the highest modality

Table 3 Frequencies of the "score division" parameter of the ISI scale $(n=120)$

\begin{tabular}{lll}
\hline ISI score division & Number & Percent \\
\hline No clinically significant insomnia & 39 & 32.5 \\
Subthreshold insomnia & 55 & 45.8 \\
Clinical insomnia moderate severity & 25 & 20.8 \\
Clinical insomnia severe & 1 & 0.8 \\
\hline
\end{tabular}

Table 4 Internal consistency and reliability of the ISI scale ( $n=$ 120)

\begin{tabular}{lll}
\hline & $\begin{array}{l}\text { Cronbach's alpha for total } \\
\text { scale with item removed }\end{array}$ & Reliability ICC (95\% Cl) \\
\hline Item 1 & 0.80 & $0.90(0.85-0.93)$ \\
Item 2 & 0.81 & $0.86(0.81-0.91)$ \\
Item $\mathbf{3}$ & 0.84 & $0.92(0.89-0.95)$ \\
Item $\mathbf{4}$ & 0.75 & $0.92(0.88-0.94)$ \\
Item 5 & 0.77 & $0.93(0.90-0.95)$ \\
Item 6 & 0.75 & $0.93(0.90-0.95)$ \\
Item 7 & 0.77 & $0.90(0.86-0.93)$ \\
Total score & 0.81 & $0.99(0.99-1.00)$ \\
\hline
\end{tabular}

ICC intra-class correlation coefficient

Kappa $=0.76$

The correlations between items and the total scale were above 0.30 and ranged from 0.31 to 0.76 . Each item can ensure the homogeneity of the measurement of the scale (Table 4).

Table 4 also showed that Cronbach's alpha reduced if we removed one of the seven items. The presence of the seven items maximized the internal consistency.
Table 5 Correlation of individual items of the Moroccan version of ISI with the total scale score

\begin{tabular}{ll}
\hline & $\begin{array}{l}\text { Pearson } \\
\text { correlations }\end{array}$ \\
\hline Item 1 & 0.62 \\
Item 2 & 0.56 \\
Item 3 & 0.54 \\
Item 4 & 0.82 \\
Item 5 & 0.74 \\
Item 6 & 0.82 \\
Item 7 & 0.74 \\
\hline
\end{tabular}

\section{Reproducibility}

Intra-observer reproducibility (test-retest) was satisfactory for the ISI scale with an intra-class correlation coefficient (ICC) $(95 \%$ CI) of 0.99 (0.989, 0.995).

The results of Table 5 show that the test-retests were in agreement for 102 people. Indeed, the kappa coefficient was 0.76 and signified a good agreement.

\section{Discussion}

The objective of our study was to translate and validate the ISI scale into Arabic Moroccan dialect. Initially, we followed the translation and cross-cultural adaptation steps recommended by the guidelines suggested by Guillemin et al. [7].

The acceptability of this scale was good, as demonstrated by the zero refusal rate and the absence of missing data.

The results of the psychometric analysis were adequate satisfactory. The Cronbach's alpha coefficient was above than 0.60 .

Items of scale are homogeneous since all of them were within the limits of the accepted discrimination threshold greater than 0.30 [14]. So, each item evaluates an attribute distinct from the construct of the measurement scale [8].

The internal consistency was satisfactory and comparable with previous studies that also found values above $0.6[4,15,16]$.

The test-retest reproducibility was good. Indeed, the intraclass correlation coefficients were all above the generally recommended threshold of 0.60 [10]. Besides, the kappa coefficient measuring the test-retest agreement signifying good to excellent agreement.

Discriminant validity was not the subject of our study since we do not have items that measure different phenomena.

Regarding the ISI scale, the Cronbach's alpha coefficient found in our study (0.83) was higher than that of 
the original version (0.74) [3], close to that of the classical Arabic version (0.84) [14], and identical to that found in the Chinese version [17]. Also, the correlations between items and scales ( 0.31 to 0.76$)$ were supported by similar correlations from the original version (0.36 to 0.67$)$ [3].

Test-retest reliability revealed that ISI results did not change significantly over time, and respondents remain highly consistent with their original survey (test).

As a result, excellent reproducibility was observed $(r$ $=0.99$ ), compatible with an ICC of 0.83 in patients with cancer [18] and 0.79 in apparently healthy adolescents [17]. Our study concluded that the ISI scale test-retest agreement was perfect, thus supporting the utility of this instrument in assessing the severity of insomnia.

This study has some limitations. We did not use another sleep assessment, and we could not study the construct validity.

\section{Conclusions}

The ISI scale was successfully translated into the Moroccan dialect. The results obtained showed that this version has adequate psychometric properties. This valid translated version could be used to screen for insomnia in the Moroccan population.

\section{Abbreviations \\ DSM: Diagnostic and Statistical Manual of Mental Disorders; ISI: Insomnia Severity Index; ICC: Inter-class correlation coefficient; CI: Confidence interval.}

\section{Acknowledgements}

We would like to express our great appreciation to Intissar Haddiya, Salah Eljabri, Yacine El Bakhat, Sara Bouqmejja, Sara Harfi For their help in the process of translation of the scales.

\section{Authors' contributions}

The three authors, $\mathrm{BO}, \mathrm{NA}$, and $\mathrm{AE}$, contributed to the conception and design of the work; the acquisition, analysis, and interpretation of the data; drafted the work; and approved the submitted version. The three authors have read and approved the final manuscript.

\section{Funding}

None.

\section{Availability of data and materials}

The authors confirm that the data supporting the findings of this study are available within the article

\section{Declarations}

\section{Ethics approval and consent to participate}

The Ethics Research Committees of the Faculty of Medicine and Pharmacy Oujda approved the study. The study was carried out under conditions that comply with the ethical recommendations of the Helsinki Declaration. We obtained signed consent from participants.

\section{Consent for publication}

The three authors (BO, NA, AE) have read and approved the manuscript.

\section{Competing interests}

The authors declare that they have no competing interests.

\section{Author details}

${ }^{1}$ Department of Psychiatry, CHU Mohammed VI, Faculty of Medicine, University Mohammed I, Hopital Arrazi, Boulevard Allal El Fassi, 60000 Oujda, Morocco. ${ }^{2}$ Department of Epidemiology, CHU Mohammed VI, Faculty of Medicine, University Mohammed I, Oujda, Morocco.

Received: 16 December 2021 Accepted: 25 January 2022

Published online: 07 February 2022

\section{References}

1. American Psychiatric Association (2013) Diagnostic and Statistical Manual of Mental Disorders, 5th edn. American Psychiatric Publishing, Arlington

2. Ohayon MM (2002) Epidemiology of insomnia: what we know and what we still need to learn. Sleep Med Rev. 6(2):97-111

3. Bastien $\mathrm{CH}$, Vallières A, Morin CM (2001) Validation of the Insomnia Severity Index as an outcome measure for insomnia research. Sleep Med. 2(4):297-307

4. Suleiman $\mathrm{KH}$, Yates BC (2011) Translating the insomnia severity index into Arabic. J Nurs Scholarsh 43(1):49-53

5. Ahmed AE (2014) Validation of the Arabic version of the Epworth sleepiness scale. J Epidemiol Glob Health 4(4):297-302

6. Suleiman KH, Yates BC, Berger AM, Pozehl B, Meza J (2010) Translating the Pittsburgh Sleep Quality Index into Arabic. West J Nurs Res 32(2):250-268

7. Guillemin DF, Bombardier C, Beaton D (1993) Cross-cultural adaptation of health-related quality of life measures: literature review and proposed guidelines. J Clin Epidemiol 46(12):1417-1432

8. Streiner DL, Norman GR, Cairney J (2015) Health Measurement Scales: a practical guide to their development and use. Oxford University Press. https://doi.org/10.1093/med/9780199685219.001.0001

9. Shrout PE, Fleiss JL (1979) Intraclass correlations: uses in assessing rater reliability. Psychol. Bull. 86(2):420-428

10. Un guide de validation transculturelle. http://instrumentspsychomet riques.mcgill.ca/instruments/guide.htm (accessed 11 July 2020)

11. Gwet KL (2014) Handbook of inter-rater reliability, 4th edition: the definitive guide to measuring the extent of agreement among raters. Advanced Analytics, LLC

12. Cronbach $L J$ (1951) Coefficient alpha and the internal structure of tests. Psychometrika 16(3):297-334

13. Nunnally J, Bernstein I (eds) (1994) Psychometric theory. McGraw-Hill, New York

14. Shapiro MB (1980) Psychometrics and psychology. Br J Psychiatry. 137(2):193-193

15. Yu DS (2010) Insomnia Severity Index: psychometric properties with Chinese community-dwelling older people. J Adv Nurs 66(10):2350-2359

16. Fernandez-Mendoza J et al (2012) The Spanish version of the Insomnia Severity Index: a confirmatory factor analysis. Sleep Med 13(2):207-210

17. Chung KF, Kan KK, Yeung WF (2011) Assessing insomnia in adolescents: comparison of Insomnia Severity Index, Athens Insomnia Scale and Sleep Quality Index. Sleep Med. 12(5):463-470

18. Savard MH, Savard J, Simard S, Ivers H (2005) Empirical validation of the Insomnia Severity Index in cancer patients. Psychooncology. 14(6):429-441

\section{Publisher's Note}

Springer Nature remains neutral with regard to jurisdictional claims in published maps and institutional affiliations. 